\title{
HYRISK: An $R$ package for hybrid uncertainty analysis using probability, imprecise probability and possibility distributions
}

\author{
Jeremy Rohmer \\ BRGM
}

\author{
Jean-Charles Manceau
}

BRGM

\author{
Dominique Guyonnet \\ BRGM-ENAG
}

\author{
Faiza Boulahya \\ BRGM
}

\author{
Didier Dubois \\ IRIT, CNRS
}

University of Toulouse

\begin{abstract}
Uncertainty analysis is an unavoidable risk assessment task (for instance for natural hazards, or for environmental issues). In situations where data are scarce, incomplete or imprecise, the systematic and only use of probabilities can be debatable. Over the last years, several alternative mathematical representation methods have been developed to handle in a more flexible manner the lack of knowledge related to input parameters of risk assessment models. This article presents an R package HYRISK dedicated to jointly handling different mathematical representation tools, namely probabilities, possibility distributions and probability functions with imprecise parameters, for the different stages of uncertainty treatment in risk assessments (i.e. uncertainty representation, propagation, sensitivity analysis and decision-making). We support the description using the case study of a dike stability analysis. The package is available at: https://cran.r-project.org/web/packages/HYRISK/index.html.
\end{abstract}

Keywords: Uncertainty quantification, sensitivity Analysis, probability, possibility, p-box, R.

\section{Introduction}

Uncertainty analysis is an unavoidable task for a proper risk assessment, like for instance for natural hazards (like volcanoes, landslides, floods, etc.), or for environmental issues (like groundwater or soil contamination), or for engineered structures. When dealing with uncertainties, two facets should be considered as outlined by several authors. See for instance for 
seismic risk: Rohmer and Baudrit (2011), for volcano risks: Marzocchi, Sandri, Gasparini, Newhall, and Boschi (2004), for environmental risk: Baudrit, Couso, and Dubois (2007). The first facet corresponds to aleatory uncertainty (also named randomness or intrinsic variability). It arises from natural variability owing to either heterogeneity or to the random character of natural processes (i.e. stochasticity). A common example of aleatory uncertainty is the variability in weather. The second facet corresponds to epistemic uncertainty and arises when dealing with partial ignorance i.e. when facing vague, incomplete or imprecise information such as limited databases and observations or imperfect modelling.

For representing aleatory uncertainty, there is a large consensus in the community about the use of probabilities under the frequentist perspective: when many observations are available, probability distributions can be inferred. In geotechnics, it is the fit of power-law to the relationship on frequency-volumes of cliff rockfalls (Dewez, Rohmer, Regard, Cnudde et al. 2013). However, for representing epistemic uncertainty, several options exist.

The systematic use of probabilities as a tool to represent epistemic uncertainties has been criticized by several authors in situations where the available data are imprecise, scarce, incomplete, vague, qualitative, etc. (Ferson 1996; Helton, Johnson, and Oberkampf 2004; Baudrit 2005; Baudrit and Dubois 2006; Baudrit et al. 2007; Dubois and Guyonnet 2011; Beer, Ferson, and Kreinovich 2013). In such highly uncertain situations, the challenge is to formulate appropriate mathematical tools and models in a quantitative manner, on the one hand, accounting for all data and pieces of information, but, on the other hand, without introducing unwarranted assumptions (Beer et al. 2013). Therefore, to overcome the shortcomings of the classical probabilistic setting, several alternative mathematical representation methods have been developed: e.g., probability boxes, possibility distributions, Dempster-Shafer structures, etc. (see an overview by Dubois and Guyonnet (2011)).

To date and to the authors' best knowledge, only a few open-source solutions exist to jointly handle different uncertainty mathematical tools (e.g., probabilities and intervals; or probabilities and fuzzy numbers) in uncertainty analysis of risk assessments (Table 1). Under the Matlab platform (The MathWorks, Inc 2012), the DSI-toolbox exists (Auer, Luther, Rebner, and Limbourg 2010) but it is restricted to Dempster-Shafer structures. More recently the more complete OpenCossan software has been developed (Patelli 2016) and handles probabilities, probability-boxes fuzzy variables and intervals at different stages of uncertainty treatment (representation, propagation, sensitivity analysis, reliability analysis, optimisation, etc.). Under the R platform (R Core Team 2017), no such computational framework exists. Available packages mainly focus on uncertainty representation, as for example the imprProbEst (Hable 2008) or dst R packages (Boivin 2015), or on data mining; e.g., clustering (evclust R package) and classification (evclass $R$ package).

The purpose of this communication is to present the R package HYRISK. The paper is organised as follows. In a first section, we summarise the main choices for the package design in order to fill the gap identified among the available open-source software solutions for uncertainty quantification with mixed uncertainty representation tools. Then, we describe the case study of a dike stability analysis (adapted from (Ferson and Tucker 2006)), which is used to describe and illustrate the HYRISK functionalities for the uncertainty quantification procedure in the subsequent sections. 


\begin{tabular}{lll}
\hline Package & Platform & Description \\
\hline DSI toolbox & Matlab & $\begin{array}{l}\text { Collection of methods for uncertainty quan- } \\
\text { tification and propagation using Dempster- } \\
\text { Shafer Theory and imprecise probabilities }\end{array}$ \\
\hline COSSAN & Matlab & $\begin{array}{l}\text { Algorithms and methods for uncertainty } \\
\text { quantification and management: numerical } \\
\text { analysis, reliability analysis, simulation, sen- } \\
\text { sitivity, optimization, robust design }\end{array}$ \\
\hline imprProbEst & $\mathrm{R}$ & $\begin{array}{l}\text { Minimum distance estimator for an impre- } \\
\text { cise probability model }\end{array}$ \\
\hline dst & $\mathrm{R}$ & $\begin{array}{l}\text { Basic probability assignments on a set of } \\
\text { possibilities (events) and combine these } \\
\text { events with Dempster's rule of combination }\end{array}$ \\
\hline evclust & $\mathrm{R}$ & $\begin{array}{l}\text { Clustering algorithms that produce a credal } \\
\text { partition }\end{array}$ \\
\hline evclass & $\mathrm{R}$ & Evidential distance-based classifier \\
\hline
\end{tabular}

Table 1: Main statistical open-source software solutions handling a combination of uncertainty representation tools

\section{Package functionalities}

To fill the gap identified in the introduction, the HYRISK package provides functions at each step of the uncertainty quantification procedure (Table 2 ).

The first step focuses on uncertainty representation. It aims at selecting the most appropriate mathematical tool to represent uncertainty on the considered parameter. The available options are: interval, possibility distribution (trapezoidal or triangular, see e.g. Baudrit and Dubois (2006)), probability distribution (normal, lognormal, beta, triangle, uniform, Gumbel or user-defined), a probability distribution with imprecise parameters, i.e. a family of parametric probability distributions represented by a p-box (Ferson, Kreinovich, Ginzburg, Myers, and Sentz 2002). For the sake of clarity, we use the generic term imprecise probability to designate such a uncertainty representation tool. The procedure in HYRISK first uses the CREATE_INPUT function to define the input variables (imprecise, random or fixed); for instance by setting the values of the bounds of the interval, the mean and the standard deviation of a normal probability distribution, etc. Second, the CREATE_DISTR function assigns the corresponding distribution (probability or possibility) to each uncertain input previously defined. A visualisation function PLOT_INPUT has also been implemented to handle the different representation forms. This is further described in Section 4.

The second step aims at conducting uncertainty propagation, i.e. evaluating the impact of the uncertainty pervading the input on the outcome of the risk assessment model. To do so, the main function is PROPAG, which implements the Monte-Carlo-based algorithm of (Baudrit et al. 2007) for jointly handling possibility and probability distributions and the algorithm of (Baudrit, Dubois, and Perrot 2008) for jointly handling possibility, probability distributions and p-boxes. Different options for summarizing and visualizing the results of this step are available, which are fully described in Section 5 .

The last step focuses on sensitivity analysis, i.e. on the study of how uncertainty in the output 


\begin{tabular}{|c|c|c|}
\hline Step & Function & Description \\
\hline Uncertainty Representation & CREATE_INPUT & $\begin{array}{l}\text { Defines the input variables (imprecise, ran- } \\
\text { dom or fixed). Five types are accounted for: } \\
\text { Probability distribution ; Possibility distri- } \\
\text { bution (either trapezoidal or triangular); In- } \\
\text { tervals; Fixed scalar value; Imprecise proba- } \\
\text { bility distributions (p-boxes) }\end{array}$ \\
\hline Uncertainty Representation & CREATE_DISTR & $\begin{array}{l}\text { Assigns the corresponding distribution } \\
\text { (probability or possibility) to each uncertain } \\
\text { input based on the input definition using } \\
\text { CREATE_INPUT }\end{array}$ \\
\hline Uncertainty Representation & PLOT_INPUT & Plots the input variables \\
\hline Uncertainty Propagation & PROPAG & $\begin{array}{l}\text { Conducts the joint propagation of probabil- } \\
\text { ity, imprecise probability and possibility dis- } \\
\text { tributions (or intervals) }\end{array}$ \\
\hline Uncertainty Propagation & PLOT_CDF & $\begin{array}{l}\text { Creates a plot summarizing the uncertainty } \\
\text { propagation in the form of a pair of lower } \\
\text { and upper Cumulative Distribution Func- } \\
\text { tion CDFs }\end{array}$ \\
\hline Uncertainty Propagation & SUMMARY_1CDF & $\begin{array}{l}\text { Summarizes the uncertainty propagation re- } \\
\text { sults in the form of a single CDF via the } \\
\text { weighting average approach of (Dubois and } \\
\text { Guyonnet 2011) }\end{array}$ \\
\hline Uncertainty Propagation & QUAN_INTERVAL & $\begin{array}{l}\text { Summarizes the uncertainty propagation re- } \\
\text { sults in the form of an interval of quantiles } \\
\text { at a given level }\end{array}$ \\
\hline Uncertainty Propagation & UNCERTAINTY & $\begin{array}{l}\text { Summarizes the uncertainty propagation re- } \\
\text { sults in the form of a global indicator corre- } \\
\text { sponding to the area between the upper and } \\
\text { lower CDFs }\end{array}$ \\
\hline Sensitivity Analysis & PINCHING_fun & $\begin{array}{l}\text { Pinches an imprecise variable to a fixed value } \\
\text { following (Ferson and Tucker 2006) }\end{array}$ \\
\hline Sensitivity Analysis & SENSI_PINCHING & $\begin{array}{l}\text { Conducts a sensitivity analysis using the } \\
\text { pinching approach of (Ferson and Tucker } \\
\text { 2006) }\end{array}$ \\
\hline
\end{tabular}

Table 2: Overview of the main functions implemented in the HYRISK package at each step of the uncertainty treatment procedure.

of a model (numerical or otherwise) can be apportioned to different sources of uncertainty in the model input (Saltelli, Ratto, Andres, Campolongo, Cariboni, Gatelli, Saisana, and Tarantola 2008). The approach, based on the pinching method of Ferson and Tucker (2006), is implemented using the PINCHING_fun and SENSI_PINCHING functions.

\section{Case study}




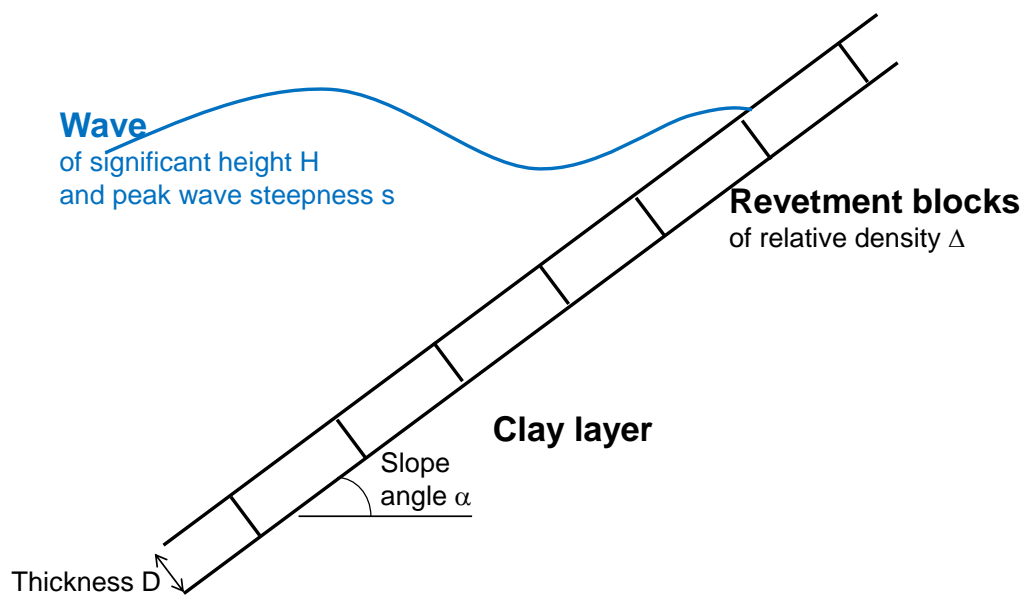

Figure 1: Schematic representation (plane view) of the dike (of slope angle $\alpha$ ) made of masonry revetment blocks (with characteristics D and $\Delta$ ) subject to wave actions (with characteristics $H$ and $s$ ). Adapted from (Ferson and Tucker 2006).

In this section, we present the study used to describe and illustrate the functionalities of the HYRISK package. This case study is focused on the stability analysis of a dike as described by Ferson and Tucker (2006). The dike has revetments made of masonry blocks subject to wave action as depicted schematically in Fig. 1.

The stability is estimated as the difference between the dike strength minus the stress acting on it as follows:

$$
Z=\text { strength }- \text { stress }=\Delta \cdot D-\frac{H \cdot \tan (\alpha)}{\cos (\alpha) \cdot M \cdot s^{0.5}}
$$

where $\Delta$ is the relative density of the revetment blocks, $D$ is their thickness, $\alpha$ is the slope of the revetment. The wave characteristics are the significant wave height $H$, and the offshore peak wave steepness $s$. The factor $M$ reflects the risk analyst's vision on the uncertainty related to the model itself, i.e. its ability to reproduce reality.

If $Z \geq 0$, the dike is stable (the strength is greater than the stress); unstable otherwise. The study is focused on the estimate of the probability for $Z$ to become negative, which is considered a measure of the dike reliability.

\section{Step 1: Uncertainty representation}

As described in the introduction, depending on the available data and information and on the nature of the model parameter, the most appropriate mathematical tools and procedures 
should be selected (Dubois and Guyonnet 2011). The HYRISK package provides a broad range of different mathematical representation tools to handle different uncertainty situations.

\subsection{Representing imprecision}

The first situation corresponds to an imprecise model parameter, i.e. a parameter whose value is ill- known, meaning that the uncertainty is purely epistemic and if we had complete information, we could expect this imprecision to drop to zero. The simplest tool to mathematically represent this imprecision is the interval, which is defined by a lower and an upper bound. In the dike example, this is the case for the revetment density $\Delta$ and the thickness $D$, which are respectively represented by $[1.60,1.65]$ and $[0.68,0.72]$ meters.

Yet, the risk analyst may provide in some cases, more information by expressing preferences inside this interval, i.e. the interval representation can be refined, see e.g., (Baudrit et al. 2007; Beer et al. 2013). This is the case for the slope angle $\alpha$. The risk analyst is certain that the value for $\alpha$ is located within the interval $[0.309,0.328]$ radians. However, according to a few measurements and his/her own experience, the analyst may be able to judge that the value 0.318 is the most likely. Using both pieces of information, the imprecision on $\alpha$ can be represented by a triangular possibility distribution. Such a distribution can be seen as a consonant set of intervals each with a level of confidence (Dubois and Prade 1988), which represents the analyst's preference and ranges between confidence one (assigned to the certain interval $[0.309,0.328]$ referred to as the support such that values located outside this interval are considered impossible) and confidence zero (assigned to the most likely value 0.318 referred to as the core of the possibility distribution).

The afore-described situation is also valid for factor $M$ since this factor reflects the confidence of the analyst on the model uncertainty (i.e. its ability to reproduce real situations), and it is subjectively defined. Yet, instead of using a unique value for defining the core (as for slope angle $\alpha$ ), the risk analyst prefers here to provide an interval so that the imprecision is in this case represented by a trapezoidal possibility distribution. Figure 2 provides some illustrations of the triangle and trapezoidal possibility distributions. The representation of the afore-described imprecision situations is achieved using the following $\mathrm{R}$ code.

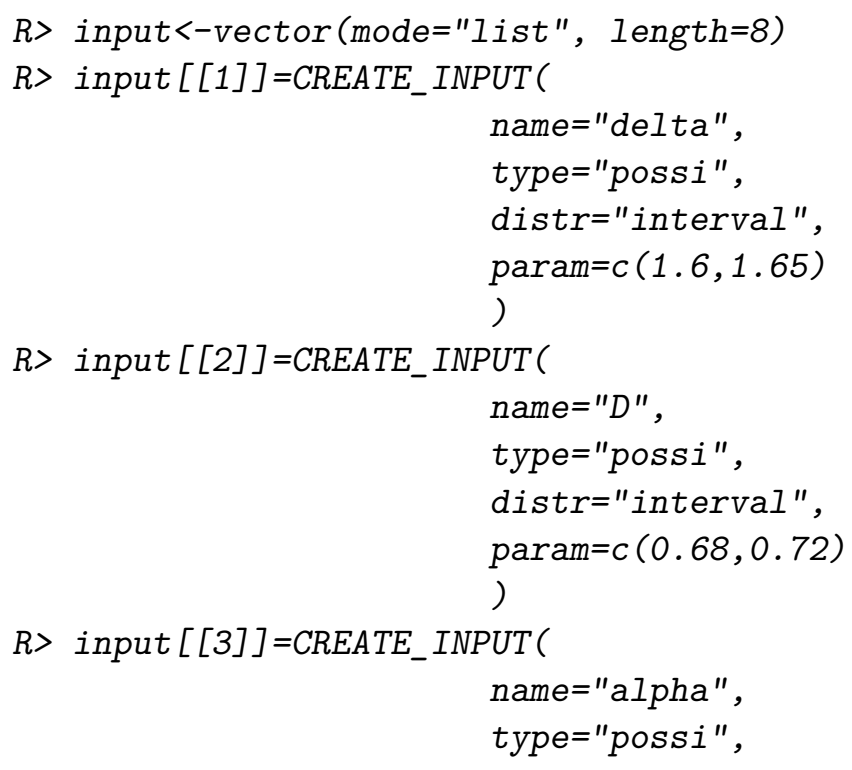




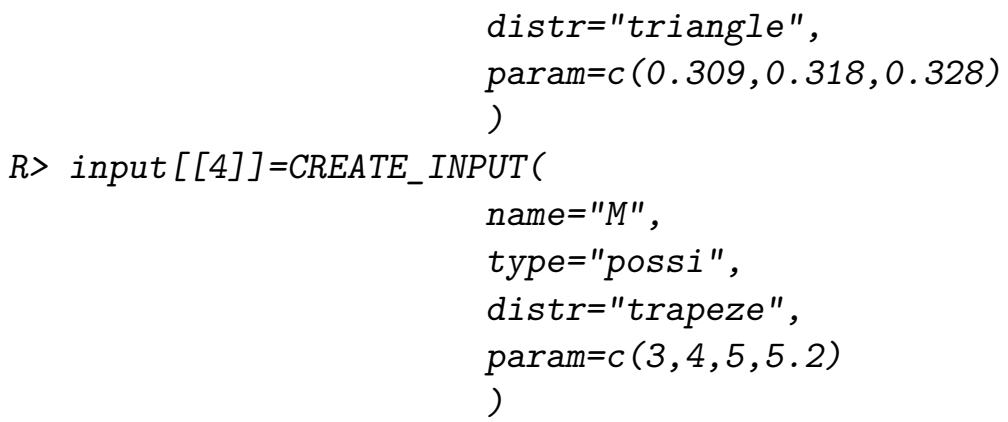

\subsection{Representing randomness}

To represent the random nature of model parameters (stochasticity), probabilities are the most appropriate representation tools; for instance by inferring the underlying statistical law. In the dike example, the wave characteristics $(s$ and $H$ ) are random variables by nature since they reflect the stochastic nature of the processes generating the waves (related to weather, seasonality and the chance of storm events, etc.). These are respectively represented by a Normal and a Weibull probability distribution. In the HYRISK package a series of different probability distribution laws are available (Normal, log-Normal, Triangular, Uniform, Beta, Gumbel) but the user can also easily define a new model (by specifying the quantile and the random sampling function).

Yet, when too few observations are available, the inference of a statistical law may be unfeasible in which case the parameters of an appropriate probability distribution may be difficult to estimate and those parameters are associated with imprecision. This is the case for the Weibull distribution of $H$ used in this example, whose shape and scale parameter $(k, \lambda)$ values cannot be precisely known due to observation scarcity. This means that there is not a unique Weibull distribution to represent the randomness of $H$, but a whole set of laws, which may be applied given the imprecision on the afore-described parameters. Two intervals for $k$ and $\lambda,[10,12]$ and $[1.2,1.5]$, are used to represent this imprecision. The ill-known Weibull probability distribution is then represented by a family probability distributions termed p-box (Ferson et al. 2002), which is bounded by a lower and an upper distribution determined by the intervals on the shape and scale parameters. The corresponding p-box is illustrated in Fig. 2.

The representation of the afore-described randomness and ill-known randomness situations is conducted using the following $\mathrm{R}$ code.

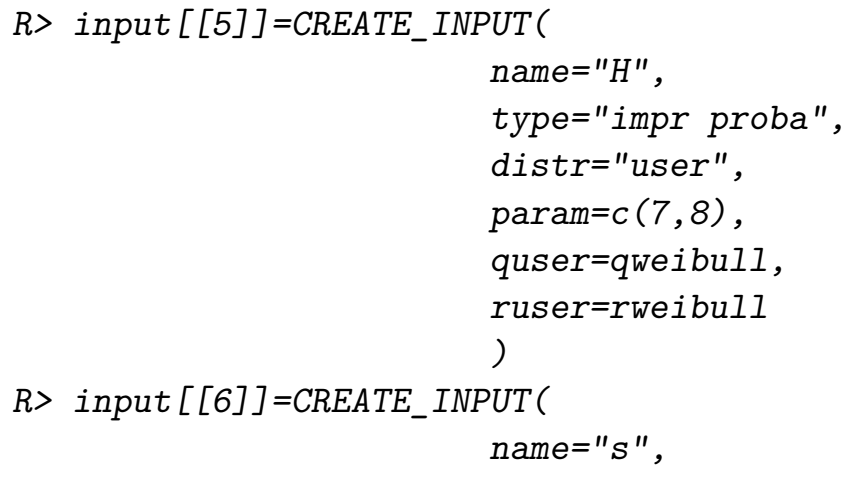




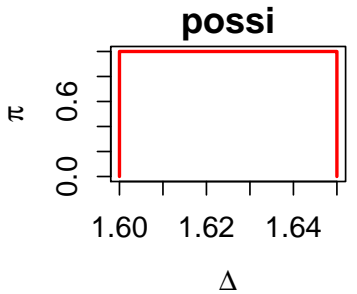

$\Delta$

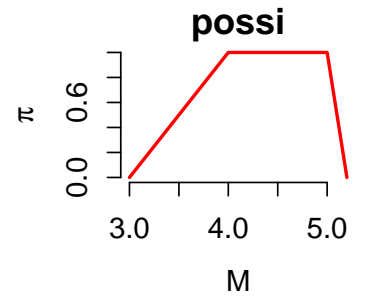

possi

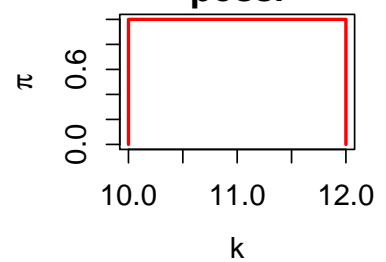

possi

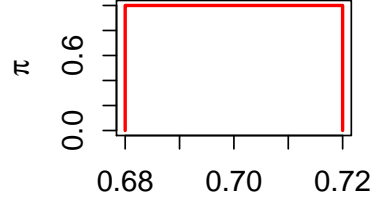

D

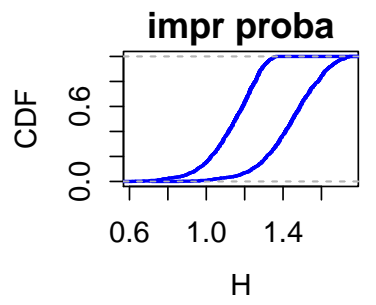

possi

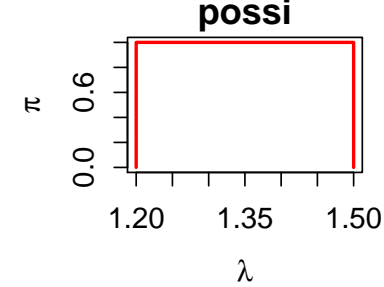

r

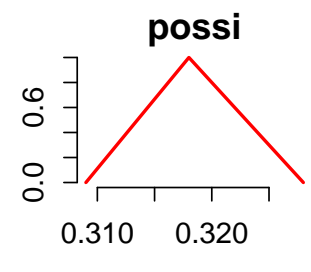

$\alpha$

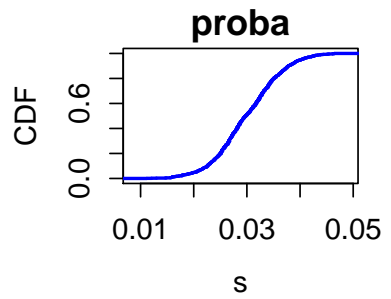

Figure 2: Uncertainty representation of the input parameters of the dike stability case. The terms proba, possi and impr proba respectively designate probability distribution, possibility distribution, and imprecise probability (i.e. p-box).

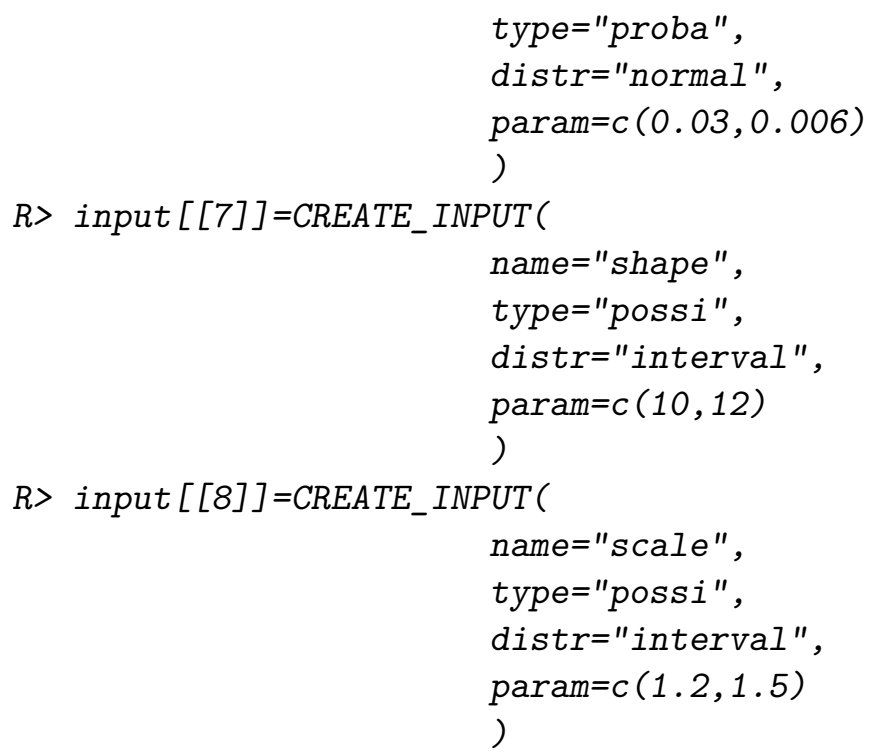

Similarly to the situation described for representing imprecision, the user may also provide a possibility distribution to represent the uncertain knowledge on the parameters of the considered imprecise probability distribution following the approach of (Baudrit et al. 2008). Table 3 summarizes the assumptions on the uncertainty representation (represented in Fig. 2) for the dike example. 


\begin{tabular}{|c|c|c|c|}
\hline Parameter & Symbol & Uncertainty type & Representation \\
\hline Significant wave height & $H$ & Randomness & $\begin{array}{l}\text { p-box of type Weibull with } \\
\text { imprecise shape and scale }\end{array}$ \\
\hline Weibull scale & $\lambda$ & Imprecision & Interval $[1.2,1.5]$ \\
\hline Weibull shape & $k$ & Imprecision & Interval $[10,12]$ \\
\hline Peak wave steepness & $s$ & Randomness & $\begin{array}{l}\text { Normal (Gaussian) prob- } \\
\text { ability distribution with } \\
\text { mean }=0.040 \text { and standard } \\
\text { deviation }=0.0055\end{array}$ \\
\hline Revetment density & $\Delta$ & Imprecision & Interval $[1.60,1.65]$ \\
\hline Revetment thickness & $H$ & Imprecision & Interval $[0.68,0.72]$ \\
\hline Slope angle & $\lambda$ & Imprecision & $\begin{array}{l}\text { Triangular } \\
\text { distribution of } \begin{array}{r}\text { possibility } \\
\text { support }\end{array} \\
{[0.309,0.328] \text { and core } 0.318}\end{array}$ \\
\hline Expert-defined factor & $M$ & Imprecision & $\begin{array}{l}\text { Trapezoidal possibility distri- } \\
\text { bution of support }[3,5.2] \text { and } \\
\text { core }[4,5] \text {. }\end{array}$ \\
\hline
\end{tabular}

Table 3: Assumptions for uncertainty representation in the dike case.

\section{Step 2: uncertainty propagation}

\subsection{Procedure}

This step aims at evaluating the uncertainty on the model output given the uncertainty in the model input parameters. Different options are available:

- When using only probabilities, a classical approach can rely on Monte-Carlo random sampling.

- When probability and possibility are used, HYRISK implements the hybrid Monte-Carlo scheme developed by Baudrit, Couso, and Dubois (2007), which combines Monte-Carlo random sampling of probability distributions and interval analysis for possibility distributions. Details of the algorithm are provided in Appendix A. This can be used following two assumptions for the fuzzy interval analysis: (1) the sampling of the possibility distributions is done randomly and independently for each imprecise parameter (algorithm named IRS Independent Random Sampling method, see (Baudrit et al. 2007) and further described in Appendix A) or (2) the sampling is deterministic and the fuzzy interval analysis is done at the same level for all imprecision parameters (algorithm termed HYBRID described by Guyonnet, Bourgine, Dubois, Fargier, Come, and Chilès (2003) and further developed by Baudrit (2005)).

- When p-boxes are also present (and especially when possibility distributions are used to represent the uncertain knowledge on the parameters of the considered imprecise probability distribution), the sampling strategy developed by Baudrit, Dubois, and Perrot (2008) and further exploited by Pedroni, Zio, Ferrario, Pasanisi, and Couplet (2013) is used (see Appendix B); 
- When imprecision on the parameters of the probability distributions are also represented by probability distributions (even though it would mix randomness with imprecision, see discussion by Baudrit, Dubois, and Perrot (2008), the propagation is conducted within a 2D Monte-Carlo scheme (e.g., (Kentel and Aral 2005)).

Since the hybrid algorithms ((Baudrit et al. 2007, 2008) are based on interval analysis, several algorithm options for solving the constrained optimization problem are available, namely:

- When the monotonicity of the input-output relationship is known for each variable, others being set at fixed values, (either increasing or decreasing), this information can be passed during the definition of the input (using the argument monoton of the function CREATE_INPUT). The optimisation problem is then solved by a combinational approach using only the values at the interval endpoints.

- When the monotony of the input-output relationship is unknown, the quasi-Newton method Limited-memory BFGS (e.g., Nocedal (1980)) is used with the optimr R package. Due to the sensitivity of the quasi-Newton algorithm to the initial best guest solution (vector of initial values for the parameters for which optimal values are to be found), a version with randomly generated multiple initial starting solutions is also provided.

- A more generic algorithm is also provided, which relies on the genetic algorithm as implemented in the rgenoud $\mathrm{R}$ package, see Mebane11.

For instance, the propagation in the dike stability case is done using the IRS algorithm with $N=1,000$ Monte-Carlo random samples and the risk assessment function (denoted here FUN and defined in Sect. 3) as follows:

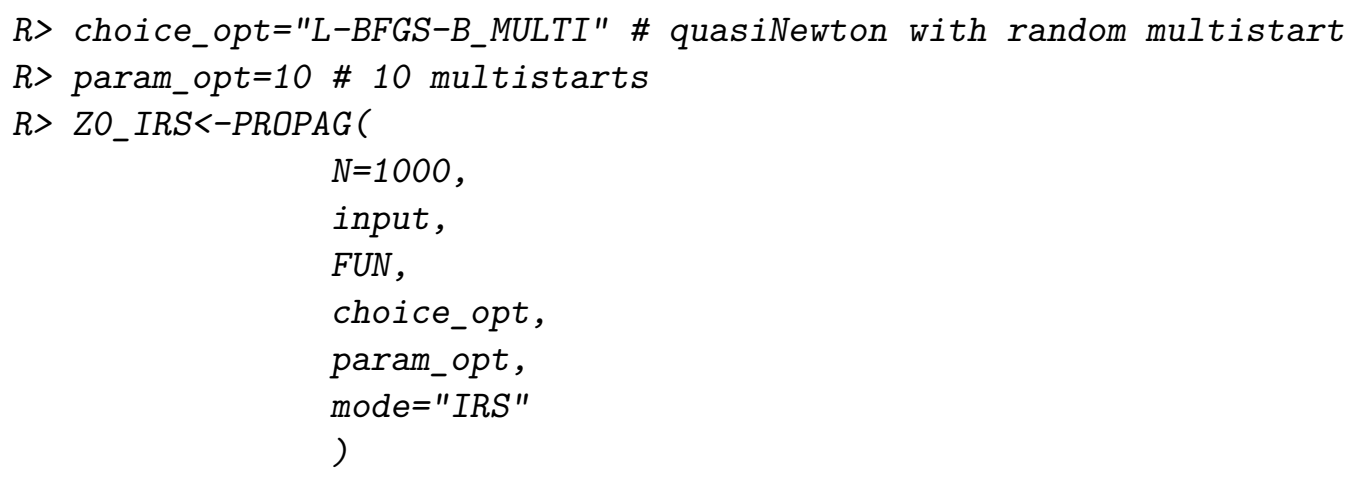

\subsection{Post-processing of the results for decision-making}

The output of the propagation procedure then takes the form of $\mathrm{N}$ random intervals of the form $\left[\underline{Z}_{k}, \bar{Z}_{k}\right]$, with $k=1, \ldots, N$. This information can be summarized in the form of a pair of upper and lower cumulative probability distributions (CDFs), in the form of a p-box which is closely related to upper and lower probabilities of Dempster (Dempster 1967), and belief functions of Shafer (Shafer et al. 1976) as proposed by Baudrit, Couso, and Dubois (2007). Figure 3 provides the output of the propagation phase using the PLOT_CDF function. 


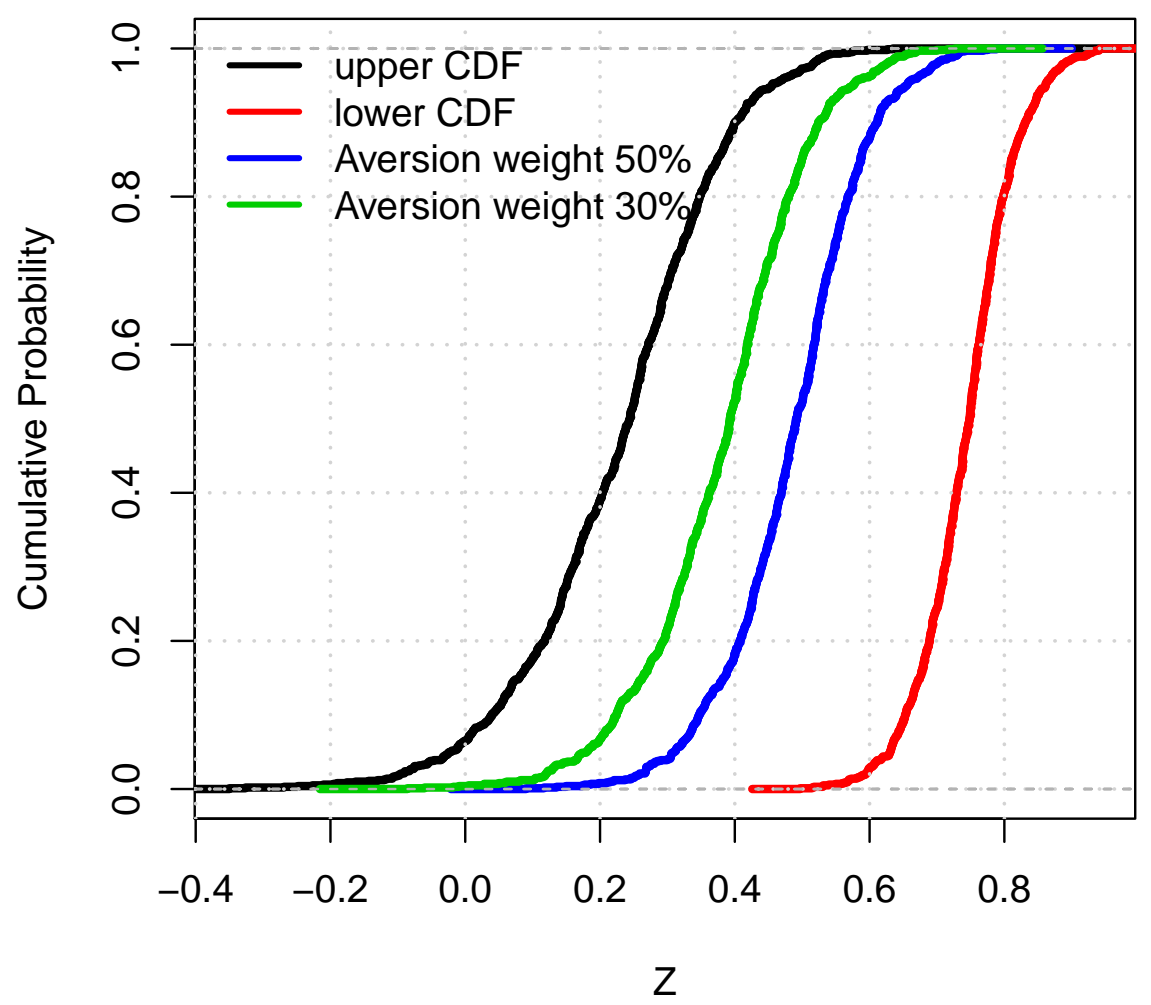

Figure 3: Results of the hybrid uncertainty propagation step for the dike stability case; the black and red lines respectively represent the lower and upper probability distribution, which defines the p-box related to the uncertainty on $\mathrm{Z}$. The green and blue lines respectively represent the probability distribution determined by a weighing averaging of both bounds, here using a weight of 30 and 50\% (following the approach of (Dubois and Guyonnet 2011)).

The functionalities of HYRISK enable to summarise the p-box of Figure 4 depending on the statistical quantity of interest supporting the decision making process.

- If the interest is the probability of $\mathrm{Z}$ being below the decision threshold at zero, the PROBA_INTERVAL function provides the corresponding upper and lower bound on this probability. In the dike case, this is comprised between $[0,7] \%$.

- If the interest is a quantile at a given level (say 75\%), the QUAN_INTERVAL function provides the corresponding interval, namely $[0.32,0.79]$ in the dike case.

- If the interest is a global measure of epistemic uncertainty affecting the whole probability distribution of $Z$, the UNCERTAINTY function computes the area within the lower and upper probability distribution, namely of 51 in the dike case. 
Finally in some situations, the analysts may be more comfortable in deriving a unique probability distribution. In order to support decision-making with a less extreme indicator than using either probability bounds, Dubois and Guyonnet (2011) proposed to weight the bounds by an index $w$, which reflects the attitude of the decision-maker to risk (i.e. the degree of risk aversion) so that the resulting distribution $F$ holds as follows:

$$
F^{-1}(x)=w \cdot \bar{F}^{-1}(x)+(1-w) \cdot \underline{F}^{-1}(x)
$$

where $\mathrm{x}$ is the quantile level ranging between 0 and 1 . This can be done using the SUMMARY_1CDF function as exemplified in Fig. 3 using two aversion weight values of respectively 30 and $50 \%$. The afore-described summary functions can be implemented as follows:

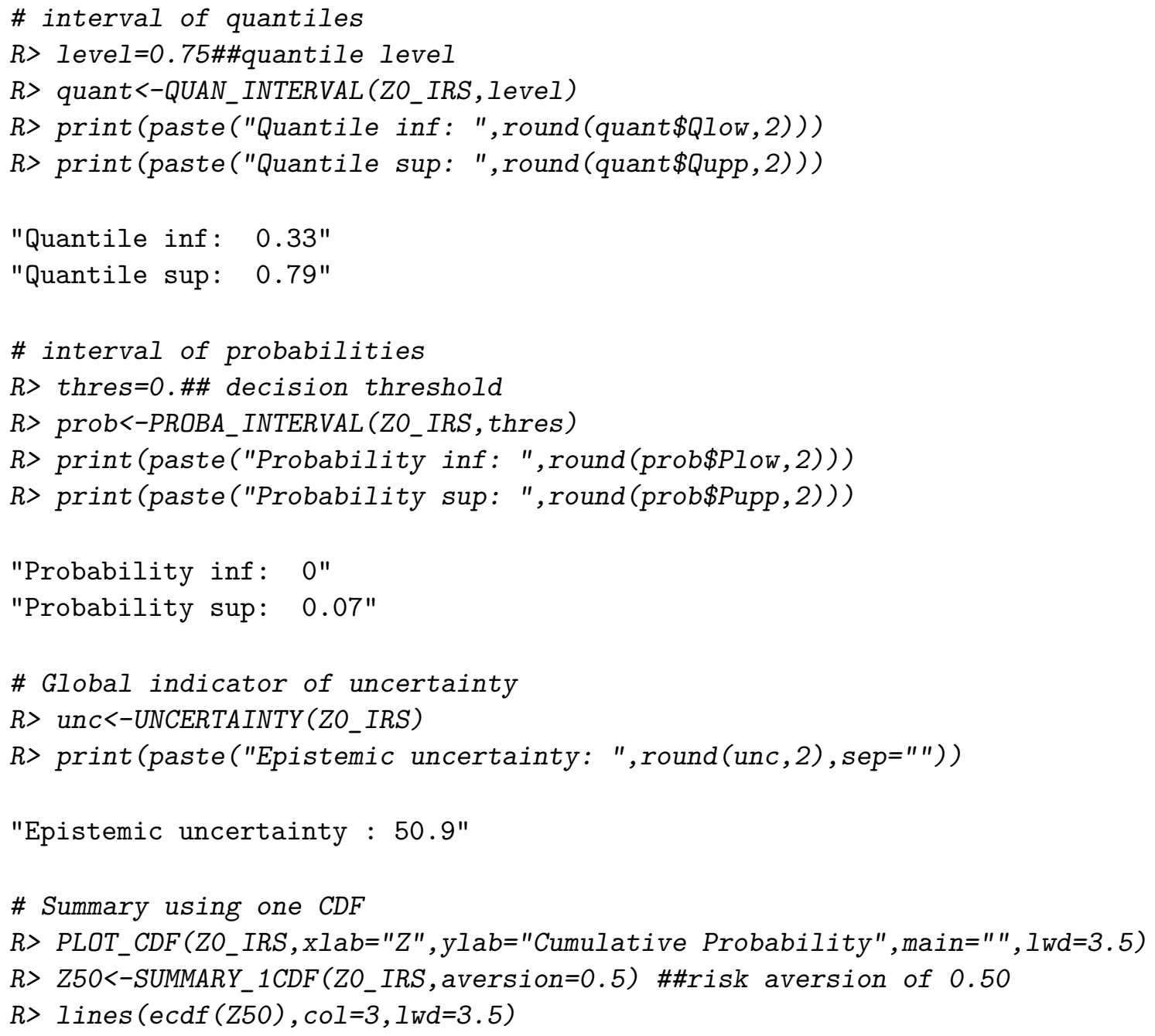

\section{Step 3: sensitivity}

When mixed representations of uncertainty are present, a practical method for sensitivity analysis is the pinching approach proposed by Ferson and Tucker (2006), which consists of 


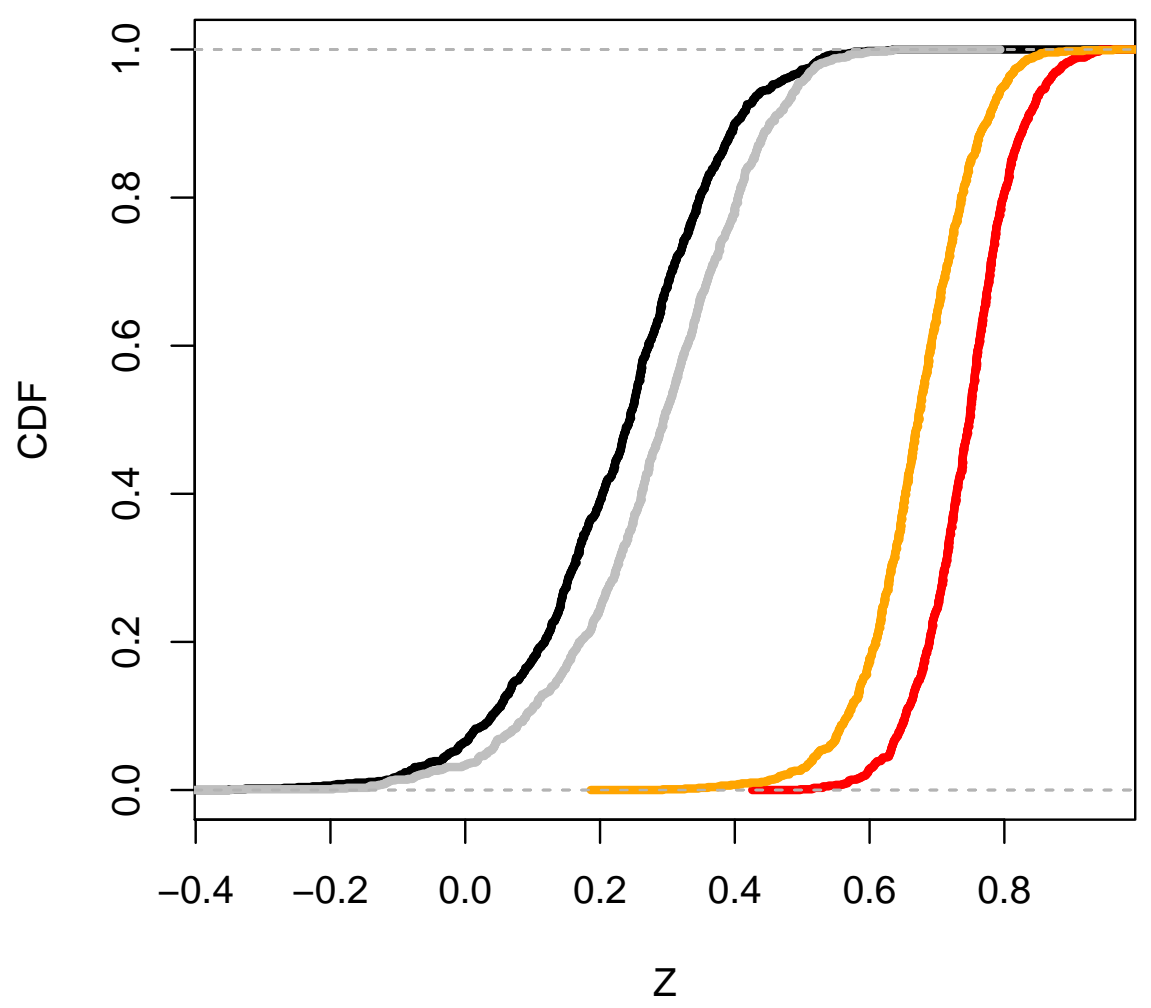

Figure 4: Lower and upper CDFs before (black and red lines) and after (grey and orange lines) pinching the scale parameter $\lambda$ to the value of 1.4.

assessing how the imprecision on the output would reduce if additional information on the input parameters were available, i.e. if imprecision in the inputs were removed / reduced. For instance, this can be performed by transforming the interval for the scale parameter $\lambda$ to a constant value (here of 1.4) or the triangular possibility distribution of $D$ to a constant value, e.g. to the core 0.7. This can be performed using the PINCHING_fun function.

Figures 4 and 5 shows the evolution of the lower and upper CDF after respectively pinching $\lambda$ and $D$.

After pinching $\lambda$ and $D$, the impact of the epistemic reduction can be summarised using the SENSI_PINCHING function in terms of:

- Reduction of the area between both CDFs, which respectively yields $\approx 24 \%$ and $\approx 13 \%$.

- Reduction of the interval of probability of exceeding the zero threshold, which respectively yields $48 \%$ and $45 \%$.

- Reduction of the interval of quantile at $75 \%$, which respectively yields $\approx 26 \%$ and $\approx 13 \%$. 


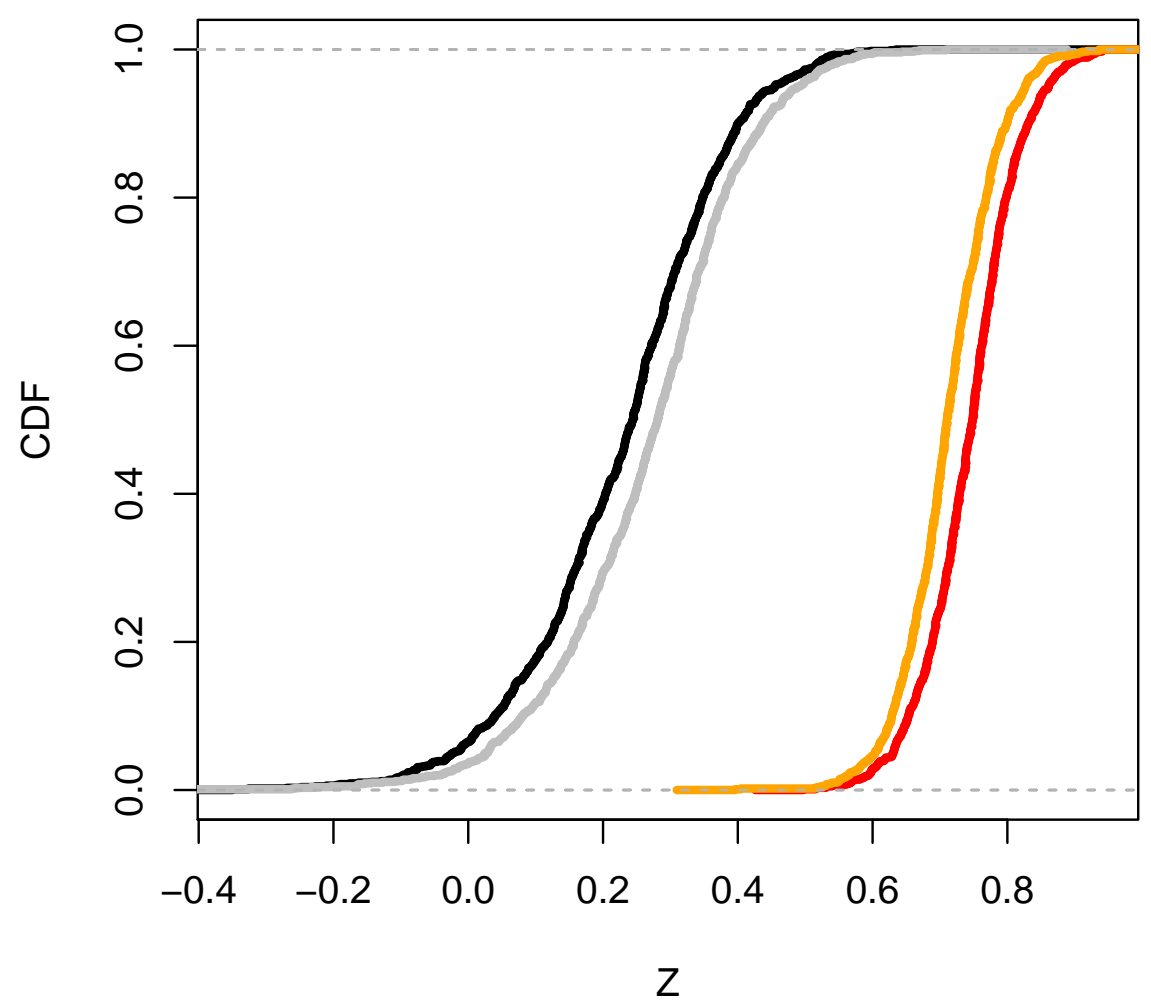

Figure 5: Lower and upper CDFs before (black and red lines) and after (grey and orange lines) pinching the block thickness $\mathrm{D}$ to the value of 0.7 .

In this example, we can conclude that $Z$ is more sensitive to the epistemic parameter $\lambda$ than to $D$ whatever the considered indicator (global, probability, quantile). The pinching procedure can be implemented as follows:

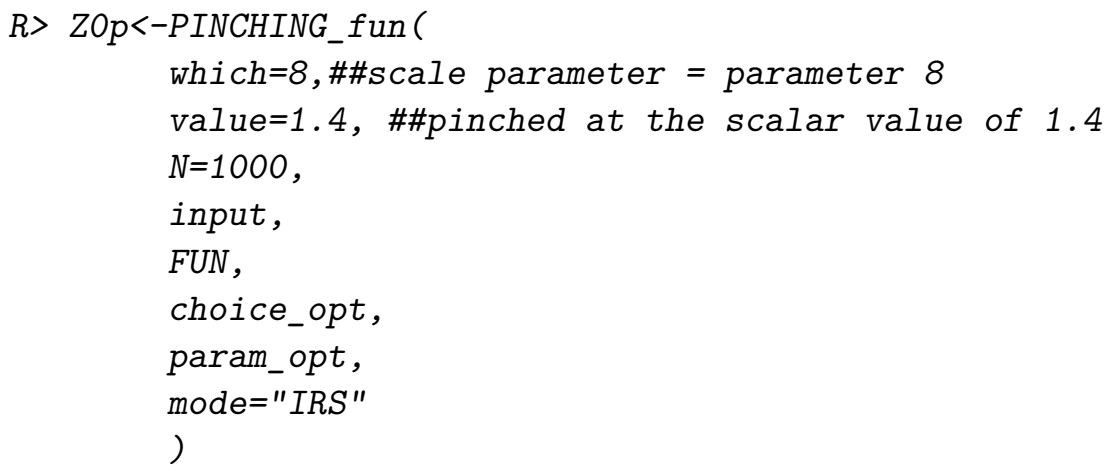




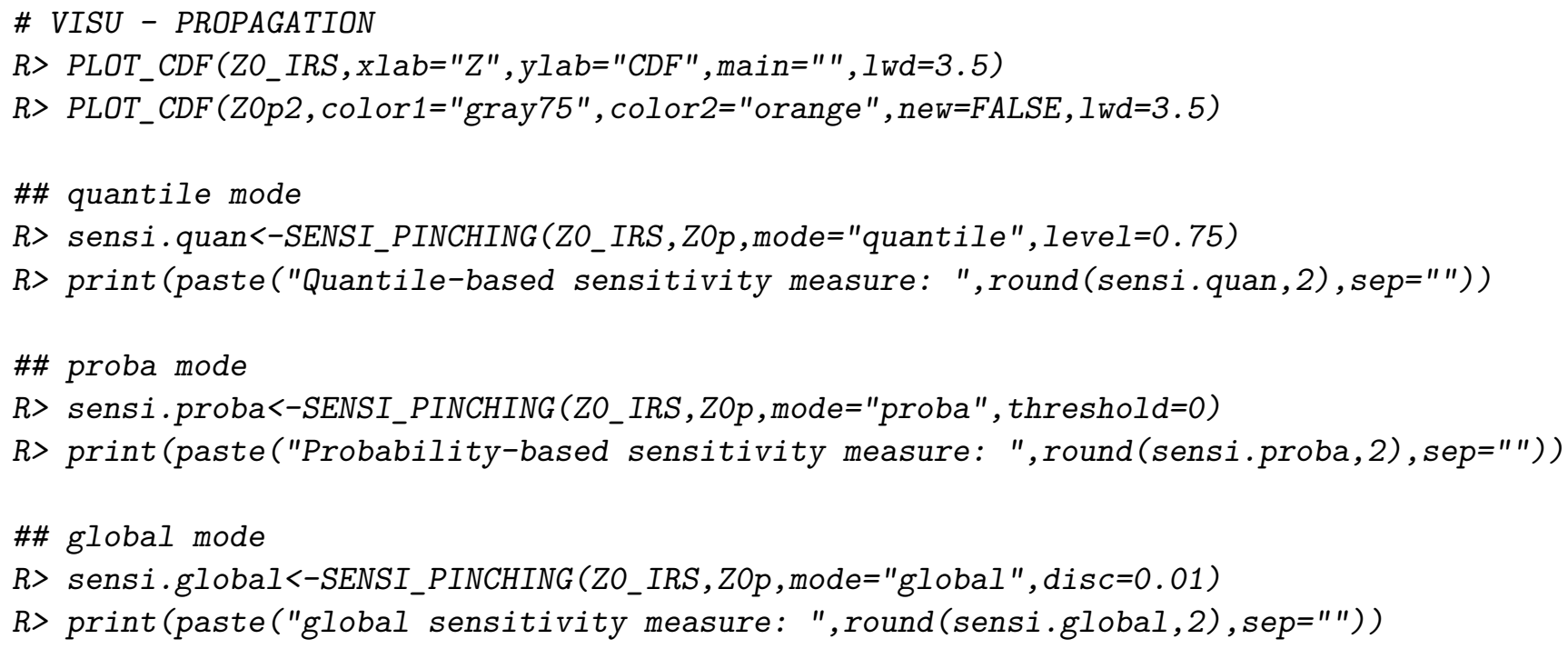

"Quantile-based sensitivity measure: 26.05"

"Probability-based sensitivity measure: 48.76"

"global sensitivity measure: 24.93"

\section{Summary and further developments}

When dealing with incomplete, scarce or imprecise data, it is of primary importance to use flexible and appropriate mathematical representation tools for accounting for all data and pieces of information, but without introducing unwarranted assumptions Beer13. The HYRISK package, described in the present communication offers, within the open source statistical software R, the capability to handle a large spectrum of different tools, namely probabilities, possibility distributions and probabilities with imprecise parameters, for the different stages of uncertainty treatment in risk assessments (i.e. uncertainty representation; propagation, sensitivity analysis and decision-making).

These functionalities were illustrated using the case study of a dike stability analysis. To date, the package focuses on situations where the risk assessment model is costless to evaluate (i.e. low computation time cost). Yet a major additional barrier to the systematic use of these techniques is their practical implementation to numerical codes. Manipulating hybrid mathematical tools goes at the expense of additional computational efforts: in a broad sense, the hybrid uncertainty propagation imposes not only to randomly sample probability distributions, through Monte-Carlo-like techniques, but also to solve constrained optimization problems. The combination with meta-modelling techniques as proposed by Lockwood, Anitescu, and Mavriplis (2012) and Eldred, Swiler, and Tang (2011) is promising. Combining the HYRISK package with such techniques (for instance by taking advantages of the suite Dice of R packages based on kriging metamodeling, (Roustant, Ginsbourger, and Deville 2012) constitutes a line for future developments. 


\section{Acknowledgments}

This work was supported in part by the Risk-R project co-funded by the French Environment Agency (ADEME; project 1472C0054). We also acknowledge financial support of BRGMfunded projects PSO Incertitudes and DEV-EXTRAPOLATE.

\section{References}

Auer E, Luther W, Rebner G, Limbourg P (2010). "A verified matlab toolbox for the dempster-shafer theory." In Workshop on the Theory of Belief Functions.

Baudrit C (2005). Représentation et propagation de connaissances imprécises et incertaines: Application à l'évaluation des risques liés aux sites et sols pollués. Ph.D. thesis, Université Paul Sabatier-Toulouse III.

Baudrit C, Couso I, Dubois D (2007). "Joint propagation of probability and possibility in risk analysis: Towards a formal framework." International Journal of Approximate Reasoning, 45(1), 82-105.

Baudrit C, Dubois D (2006). "Practical representations of incomplete probabilistic knowledge." Computational statistics $\& 3$ data analysis, 51(1), 86-108.

Baudrit C, Dubois D, Perrot N (2008). "Representing parametric probabilistic models tainted with imprecision." Fuzzy sets and systems, 159(15), 1913-1928.

Beer M, Ferson S, Kreinovich V (2013). "Imprecise probabilities in engineering analyses." Mechanical Systems and Signal Processing, 37(1), 4-29.

Boivin C (2015). dst: Using Dempster-Shafer Theory. URL https://cran.r-project.org/ web/packages/dst/index.html.

Dempster AP (1967). "Upper and lower probabilities induced by a multivalued mapping." The annals of mathematical statistics, pp. 325-339.

Dewez TJ, Rohmer J, Regard V, Cnudde C, et al. (2013). "Probabilistic coastal cliff collapse hazard from repeated terrestrial laser surveys: case study from Mesnil Val (Normandy, northern France)." Journal of Coastal Research, 65, 702-707.

Dubois D, Guyonnet D (2011). "Risk-informed decision-making in the presence of epistemic uncertainty." International Journal of General Systems, 40(02), 145-167.

Dubois D, Prade H (1988). Possibility theory. Springer.

Eldred M, Swiler LP, Tang G (2011). "Mixed aleatory-epistemic uncertainty quantification with stochastic expansions and optimization-based interval estimation." Reliability Engineering 83 System Safety, 96(9), 1092-1113.

Ferson S (1996). "What Monte Carlo methods cannot do." Human and Ecological Risk Assessment, 2(4), 990-1007. 
Ferson S, Kreinovich V, Ginzburg L, Myers DS, Sentz K (2002). Constructing probability boxes and Dempster-Shafer structures, volume 835. Sandia National Laboratories.

Ferson S, Tucker T (2006). "Sensitivity analysis using probability bounding." Reliability Engineering 83 System Safety, 91(10), 1435-1442.

Guyonnet D, Bourgine B, Dubois D, Fargier H, Come B, Chilès JP (2003). "Hybrid approach for addressing uncertainty in risk assessments." Journal of environmental engineering, $\mathbf{1 2 9}(1), 68-78$.

Hable R (2008). imprProbEst: Minimum distance estimation in an imprecise probability model.

Helton JC, Johnson JD, Oberkampf WL (2004). "An exploration of alternative approaches to the representation of uncertainty in model predictions." Reliability Engineering 83 System Safety, 85(1), 39-71.

Kentel E, Aral M (2005). "2D Monte Carlo versus 2D fuzzy Monte Carlo health risk assessment." Stochastic Environmental Research and Risk Assessment, 19(1), 86-96.

Lockwood B, Anitescu M, Mavriplis DJ (2012). "Mixed aleatory/epistemic uncertainty quantification for hypersonic flows via gradient-based optimization and surrogate models." In the 50th AIAA Aerospace Sciences Meeting Including the New Horizons Forum and Aerospace Exposition, Nashville, Tennessee, pp. 9-12.

Marzocchi W, Sandri L, Gasparini P, Newhall C, Boschi E (2004). "Quantifying probabilities of volcanic events: the example of volcanic hazard at Mount Vesuvius." Journal of Geophysical Research: Solid Earth (1978-2012), 109(B11).

Nocedal J (1980). "Updating quasi-Newton matrices with limited storage." Mathematics of computation, 35(151), 773-782.

Patelli E (2016). "COSSAN: a multidisciplinary software suite for uncertainty quantification and risk management." In Handbook of uncertainty quantification, pp. 1-69. Springer.

Pedroni N, Zio E, Ferrario E, Pasanisi A, Couplet M (2013). "Hierarchical propagation of probabilistic and non-probabilistic uncertainty in the parameters of a risk model." Computers \& Structures, 126, 199-213.

R Core Team (2017). R: A Language and Environment for Statistical Computing. R Foundation for Statistical Computing, Vienna, Austria. URL https://www.R-project.org/.

Rohmer J, Baudrit C (2011). "The use of the possibility theory to investigate the epistemic uncertainties within scenario-based earthquake risk assessments." Natural hazards, 56(3), 613-632.

Roustant O, Ginsbourger D, Deville Y (2012). "DiceKriging, DiceOptim: Two R packages for the analysis of computer experiments by kriging-based metamodeling and optimization." Journal of Statistical Software, 51, 1-55.

Saltelli A, Ratto M, Andres T, Campolongo F, Cariboni J, Gatelli D, Saisana M, Tarantola S (2008). Global sensitivity analysis: the primer. John Wiley \& Sons. 
Shafer G, et al. (1976). A mathematical theory of evidence, volume 1. Princeton university press Princeton.

The MathWorks, Inc (2012). MATLAB âĂŞ The Language of Technical Computing, Version R2012a. The MathWorks, Inc., Natick, Massachusetts. URL http://www . mathworks .com/ products/matlab/. 


\section{A. IRS algorithm with possibilities and probabilities}

Consider k random input variables $X_{i}(\mathrm{i}=1, \ldots, \mathrm{k})$, each of them associated to a cumulative probability distribution $F$, and n-k imprecise input variables $X_{i}(\mathrm{i}=\mathrm{k}+1, \ldots, \mathrm{n})$, each of them associated to a possibility distribution $\pi$. In this situation, the IRS procedure proceeds as follows. First randomly generate from uniform probability distributions, m vectors of size n: $\alpha_{i}, \mathrm{i}=1, \ldots, \mathrm{n}$, such that $0 \leq \alpha_{i} \leq 1$. For each realisation:

1. Generate $\mathrm{k}$ values for the random input variables by using the inverse function of $F_{i}$ : $x_{i}=F_{i}^{-1}\left(\alpha_{i}\right), \mathrm{i}=1, \ldots, \mathrm{k}$

2. Sample n-k intervals $I_{i}$ corresponding to the cuts of the possibility distributions with level of confidence $1-\alpha_{i}, \mathrm{i}=\mathrm{k}+1, \ldots, \mathrm{n}$

3. Evaluate the interval $[\underline{Z}, \bar{Z}]$ defined by the lower and upper bounds associated to the model output $\mathrm{Z}$ using the risk assessment model $\mathrm{G}$ using equation 3.

$$
\underline{Z}=\inf _{I}\left(G\left(x_{1}, \ldots, x_{k}, I_{k+1}, \ldots, I_{n}\right)\right) \quad \bar{Z}=\sup _{I}\left(G\left(x_{1}, \ldots, x_{k}, I_{k+1}, \ldots, I_{n}\right)\right)
$$

\section{B. IRS algorithm with possibilities, probabilities, and with p-boxes}

Consider $\mathrm{k}$ random input variables $X_{i}(\mathrm{i}=1, \ldots, \mathrm{k})$, each of them associated to a cumulative probability distribution $F, n-k$ imprecise input variables $X_{i}(\mathrm{i}=\mathrm{k}+1, \ldots, \mathrm{n})$, each of them associated to a possibility distribution $\alpha$ and $\mathrm{j}-(\mathrm{n}+\mathrm{k})$ random input variables tainted with imprecision $X_{i}(\mathrm{i}=\mathrm{n}+1, \ldots, \mathrm{j})$, each of them associated to a parametric probabilistic model with imprecise input variables. In this situation, the IRS procedure holds as follows. Randomly generate from uniform probability distributions, $\mathrm{m}$ vectors of size $\mathrm{j}: a l p h a_{i}, \mathrm{i}=1, \ldots, \mathrm{j}$, such that $0 \leq \alpha_{i} \leq 1$. For each realisation:

1. Generate $\mathrm{k}$ values for the random input variables by using the inverse function of $F_{i}$ : $x_{i}=F_{i}^{-1}\left(a l p h a_{i}\right), \mathrm{i}=1, \ldots, \mathrm{k}$;

2. Sample n-k intervals $I_{i}$ corresponding to the cuts of the possibility distributions with level of confidence: $1-\alpha_{i}, \mathrm{i}=\mathrm{k}+1, \ldots, \mathrm{n}$;

3. For each random input variables tainted with imprecision $X_{i}(\mathrm{i}=\mathrm{n}+1, \ldots, \mathrm{j})$, from the intervals $I_{\text {parameters }}$ sampled at the previous stage (2) for the imprecise parameters associated to this variable, evaluate the interval:

$$
\left[\inf _{I_{\text {parametersof } X_{i}}}\left(F_{i}^{-1}\left(\alpha_{i}\right)\right) ; \sup _{I_{\text {parametersof } X_{i}}}\left(F_{i}^{-1}\left(\alpha_{i}\right)\right)\right]
$$

4. Evaluate the interval $[\underline{Z}, \bar{Z}]$ defined by the lower and upper bounds associated to the model output $\mathrm{Z}$ using the risk assessment model $\mathrm{G}$ as follows: 
$\underline{Z}=\inf _{I}\left(G\left(x_{1}, \ldots, x_{k}, I_{k+1}, \ldots, I_{n}, I_{n+1}, \ldots, I_{j}\right)\right) \quad \bar{Z}=\sup _{I}\left(G\left(x_{1}, \ldots, x_{k}, I_{k+1}, \ldots, I_{n}, I_{n+1}, \ldots, I_{j}\right)\right)$

\section{Correspondence::}

Jeremy Rohmer

BRGM

3 av. C. Guillemin

45060 OrlÃlans Cedex 2

France

E-mail: j.rohmer@brgm.fr 